\title{
The chemistry of compact planetary nebulae ${ }^{\star}$
}

\author{
E. Josselin ${ }^{1}$ and R. Bachiller ${ }^{2}$ \\ 1 GRAAL-CC72, UMR 5024-ISTEEM, CNRS/Univ. Montpellier II, 34095 Montpellier Cedex, France \\ 2 Observatorio Astronómico Nacional (OAN), IGN, Apartado 1143, 28800 Alcalá de Henares, Spain
}

Received 29 July 2002 / Accepted 14 October 2002

\begin{abstract}
We report high-sensitivity millimetre observations of several molecular species $\left({ }^{13} \mathrm{CO}, \mathrm{HCN}, \mathrm{HNC}, \mathrm{CN} \mathrm{HCO}^{+}\right.$and $\mathrm{N}_{2} \mathrm{H}^{+}$) in a sample of compact planetary nebulae. Some species such as $\mathrm{HCO}^{+}$and $\mathrm{CN}$ are particularly abundant compared to envelopes around AGB stars or even interstellar clouds. We have estimated the following average values for the column densities ratios: $\mathrm{CN} / \mathrm{HCN} \sim 2.6, \mathrm{HCO}^{+} / \mathrm{HCN} \sim 0.5$, and $\mathrm{HNC} / \mathrm{HCN} \sim 0.4$. Thus, the chemical composition of the molecular envelopes in these compact PNe appears somewhat intermediate between the composition of proto-PNe (such as CRL 2688 or CRL 618) and well evolved PNe (such as the Ring, M4-9, or the Helix). From observations of the CO isotopomers, we have estimated that the ${ }^{12} \mathrm{C} /{ }^{13} \mathrm{C}$ ratio is in the range $10 \lesssim{ }^{12} \mathrm{C} /{ }^{13} \mathrm{C} \lesssim 40$. These values are below those expected from standard asymptotic giant branch models and suggest non-standard mixing processes. The observed molecular abundances are compared to very recent modelling work, and we conclude that the observations are well explained, in general terms, by time-dependent gas-phase chemical models in which the ionization rate is enhanced by several orders of magnitude with respect to the average interstellar value. Thus, our observations confirm that the chemistry in the neutral shells of PNe is essentially governed by the high energy radiation from the hot central stars. The complexity of the chemical processes is increased by numerous factors linked to the properties of the central star and the geometry and degree of clumpiness of the envelope. Several aspects of the PN chemistry that remains to be understood are discussed within the frame of the available chemical models.
\end{abstract}

Key words. planetary nebulae: general - ISM: molecules

\section{Introduction}

While planetary nebulae (PNe) are primarily identified from their optical emission arising in the ionized gas, they often contain a substantial amount of neutral gas which is a more direct relic of the mass lost on the asymptotic giant branch (AGB). $\mathrm{CO}$ and $\mathrm{H}_{2}$ surveys of $\mathrm{PNe}$ have revealed that a large fraction of the gas in massive PNe is in molecular form (Huggins et al. 1996; Kastner et al. 1996). This molecular component may be dominant during a large part of the evolution of the nebulae, and thus plays a prominent role in the mass distribution and the shaping of PNe.

The molecular gas in PNe is affected by the large radiation fields from the central stars which are expected to cause a dramatic effect on the chemical composition. The studies of the chemistry of the neutral gas in PNe have been very scarce so far. Cox et al. (1992) observed the evolved PNe NGC 6072 and IC 4406 at millimetre wavelengths, and detected HCN,

Send offprint requests to: E. Josselin,

e-mail: josselin@graal.univ-montp2.fr

* Based on observations carried out with the IRAM 30m telescope. IRAM is supported by INSU/CNRS (France), MPG (Germany) and IGN (Spain).
$\mathrm{HNC}, \mathrm{CN}$ and $\mathrm{HCO}^{+}$in both objects. More recently, Bachiller et al. (1997a) made a more complete study, covering an evolutionary sequence. If we except the two PPNe of their sample, five PNe were searched and detected over this large variety of molecular species: the young PN NGC 7027 and the evolved ones NGC 6720 (the Ring), M 4-9, NGC 6781 and NGC 7293 (the Helix). The molecular content of proto-PNe has also been investigated by observing some selected objects such as CRL 618 (Bujarrabal et al. 1988; Cernicharo et al. 2001), and OH 231.8+4.8 (Sánchez Contreras et al. 1997). It clearly appears from these studies that the chemical evolution is dominated by photodissociation, ion/radical-molecular reactions, and shocks. In particular, the survival of some molecules at well advanced stages of the evolution is made possible thanks to the shielding of the radiation, which is directly connected with the presence of inhomogeneities in the envelope. Very dense clumps, directly observed as cometary globules in the nearest PNe such as the Helix (Huggins et al. 1992, 2002), provide an environment which is well suited to the protection of the molecules and of its on-going chemistry. The chemistry of the PNe neutral envelopes has been modelled by several authors. The first work was done by Black (1978) who studied the neutral-ionized transition zone in an homogeneous nebula. 
Howe et al. (1992, 1994) modelled the evolution of a dense clump similar to those of the Helix nebula. More recently, Hasegawa et al. (2000) and Hasegawa \& Kwok (2001) reported detailed models of the neutral envelope of NGC 7027, and Ali et al. (2001) constructed time-dependent gas-phase models which reproduce well the molecular abundances in evolved PNe such as NGC6781, M4-9 and the Helix, observed by Bachiller et al. (1997a).

With the remarkable exception of NGC 7027, the previous chemical studies carried out so far have been mainly devoted either to rather evolved PNe (such as the Ring or the Helix) or to proto-PNe (such as CRL 618 or $\mathrm{OH} 231.8+4.8$ ). We report here on high-sensitivity observations of molecular lines in a sample of 7 compact, young or intermediate-aged $\mathrm{PNe}$, which improve our knowledge of the chemistry during the nebular development. The sample and the observations are presented in Sect. 2. An account of the results is given in Sect. 3. These results are discussed in Sect. 4 making particular emphasis on the chemistry of N-bearing compounds, on the formation of molecular ions, and on the importance of the clumpiness.

\section{Sample and observations}

\subsection{PNe sample}

Our sample is made of 6 compact $\mathrm{PNe}$ covering a wide range of evolutionary status and whose molecular chemistry has not been studied up to now. These are BV 5-1 (see Josselin \& Bachiller 2001 for its CO content), K 3-94, K 3-24 (Josselin et al. 2000), M 1-13, M1-17 and IC 5117 (Huggins et al. 1996 and references therein). Although it was already observed by Bachiller et al. (1997a), as representative of young PNe, we also observed NGC 7027 for comparison and calibration purposes. Table 1 lists the main properties of the observed nebulae: the most usual name (Col. 1), standard galactic name (2), kinematic distance - based on the simple approximation for the velocity structure of the Galactic disk of Burton (1974) - (3), optical size of the ionized nebula (4), radial and expansion velocities measured in the $\mathrm{CO}(2-1)$ line $(5,6)$, integrated intensity of the $\mathrm{CO}(2-1)$ line (7), mass of the molecular envelope (8), and ratio $M_{\mathrm{m}} / M_{\mathrm{i}}$ between the molecular mass and the ionized mass (9), which is a good indicator of the evolutionary stage, almost independent of the distance (Huggins et al. 1996). Concerning the distance, we have preferred the kinematic estimate over the statistical estimate, since we believe that the former is more precise in these cases (see Josselin et al. 2000 for details). Nevertheless, for NGC 7027, the best studied object in the sample, in accordance with previous works, we have assumed the standard estimate of $0.7 \mathrm{kpc}$ (see e.g. Huggins et al. 1996). In the case of M 1-13, M 1-17 and IC 5117 the values of $M_{\mathrm{m}}$ and $M_{\mathrm{m}} / M_{\mathrm{i}}$ have been re-scaled from those given by Huggins et al. (1996) according to our choice of distance $\left(M_{\mathrm{m}} \propto \text { distance }^{2} \text { and } M_{\mathrm{m}} / M_{\mathrm{i}} \propto \text { distance }^{-1 / 2}\right)^{1}$.

\footnotetext{
${ }^{1}$ The ionized mass is derived from the $\lambda 6 \mathrm{~cm}$ emission and the angular nebular size, resulting in a distance ${ }^{5 / 2}$ dependance, as shown by Gathier (1987).
}

\subsection{Observations}

The observations were carried out with the IRAM 30-m radiotelescope on Pico Veleta (near Granada, Spain) in July 1999. The search for molecular emission was made simultaneously in four lines. We used SIS receivers operating in the bands around $\lambda 3 \mathrm{~mm}$ and $\lambda 1 \mathrm{~mm}$ with typical system temperatures of 150 and $300 \mathrm{~K}$, respectively. The half-power beam width of the telescope is $22^{\prime \prime}$ and $10^{\prime \prime}$ at 3 and $1 \mathrm{~mm}$ respectively. The spectrometers were $256 \times 1 \mathrm{MHz}$ filter banks providing a velocity resolution of 2.6 and $1.3 \mathrm{~km} \mathrm{~s}^{-1}$ at 3 and $1 \mathrm{~mm}$, respectively. The observations were done by wobbling the secondary mirror to a distance of $2^{\prime}$ from the source. The pointing accuracy, checked every hour, was within $3^{\prime \prime}$. Calibration was achieved with a chopper wheel. The intensities given hereafter are in units of main beam brightness temperature.

\section{Results}

\subsection{Spectra}

Spectra obtained toward the sample of PNe are shown in Fig. 1. Table 2 lists the line integrated intensities and upper limits derived from such spectra. The integrated intensities for NGC 7027 are generally found to be compatible with those previously reported by Bachiller et al. (1997a). A significant difference was only found for the ${ }^{13} \mathrm{CO}(2-1)$ line, for which we report an intensity higher by a factor of $30 \%$. We caution that slight pointing errors can result in important differences in the line intensities of such compact objects, and we believe that the observations reported here were made with better pointing accuracy.

$\mathrm{HCO}^{+}$is detected in all the $\mathrm{PNe}$ of our sample. At least one of the N-bearing molecules we looked for $(\mathrm{HCN}, \mathrm{HNC}, \mathrm{CN})$ is also detected in every $\mathrm{PN}$, the three molecules being detected in $\mathrm{K}$ 3-94 and M 1-17. The HNC(1-0) emission is weak, and we only have at best tentative detections. ${ }^{13} \mathrm{CO}$ is detected in BV 5-1, M 1-17, K 3-24, IC 5117 and NGC 7027.

We also observed the $\mathrm{N}_{2} \mathrm{H}^{+}(1-0)$ line in the sample. The first detection of this line in a circumstellar envelope was reported by Cox et al. (1993) in NGC 7027 (see their Table 1). A high-sensitivity spectrum taken toward NGC 7027 is shown in Fig. 2, and its intensity is found to be consistent with that reported by Cox et al. (1993). This line was not detected in any other PN. The detection limits are reported in Table 2.

\subsection{Column densities and their evolution}

Estimates of the column densities have been made under the usual assumptions of LTE and optically thin emission. The excitation temperature was assumed to be uniform and equal to $25 \mathrm{~K}$, which is the kinetic temperature deduced from $\mathrm{CO}$ observations in a few other nebulae (see Bachiller et al. 1993). When the line was not detected, we used three times the upper limits to the integrated intensities given at a $1 \sigma$ level in Table 2 to derive upper limits in the corresponding column densities. The $\mathrm{CN}$ column densities have been calculated from the $\mathrm{CN}(2-1)$ line intensities, since this line is expected to be optically thinner than the $\mathrm{CN}(1-0)$ line and, in addition, the 
Table 1. Properties of the sample of PNe.

\begin{tabular}{lcccccccc}
\hline \hline Name & PN G & $\begin{array}{c}D_{\mathrm{K}} \\
(\mathrm{kpc})\end{array}$ & $\begin{array}{c}\text { size } \\
\left({ }^{\prime \prime}\right)\end{array}$ & $\begin{array}{c}V_{\mathrm{lsr}} \\
\left(\mathrm{km} \mathrm{s}^{-1}\right)\end{array}$ & $\begin{array}{c}V_{\text {exp }} \\
\left(\mathrm{km} \mathrm{s}^{-1}\right)\end{array}$ & $\begin{array}{c}I_{12} \mathrm{CO}(2-1) \\
\left(\mathrm{K} \mathrm{km} \mathrm{s}^{-1}\right)\end{array}$ & $\begin{array}{c}M_{\mathrm{m}} \\
\left(10^{-2} M_{\odot}\right)\end{array}$ & $M_{\mathrm{m}} / M_{\mathrm{i}}$ \\
\hline BV 5-1 & $119.3+00.3$ & 5.5 & 20 & -73 & 10 & 9.7 & 2.8 & 0.3 \\
K 3-94 & $142.1+03.4$ & 5.5 & 7 & -69 & 16 & 14.1 & 3.4 & 0.2 \\
M 1-13 & $232.4-01.8$ & 2.0 & 10 & 27 & 18 & 22.2 & 25.4 & 1.8 \\
M 1-17 & $228.8+05.3$ & 2.5 & 3 & 28 & 39 & 66.2 & 6.1 & 14.0 \\
K 3-24 & $048.7+02.3$ & 3.5 & 6 & 44 & 24 & 23.2 & 2.1 & 0.2 \\
IC 5117 & $089.8-05.1$ & 3.0 & 1 & -11 & 14 & 19.6 & 24.5 & 2.1 \\
NGC 7027 & $084.9-03.4$ & 0.7 & 14 & 26 & 23 & 278.0 & 22.0 & 9.4 \\
\hline
\end{tabular}
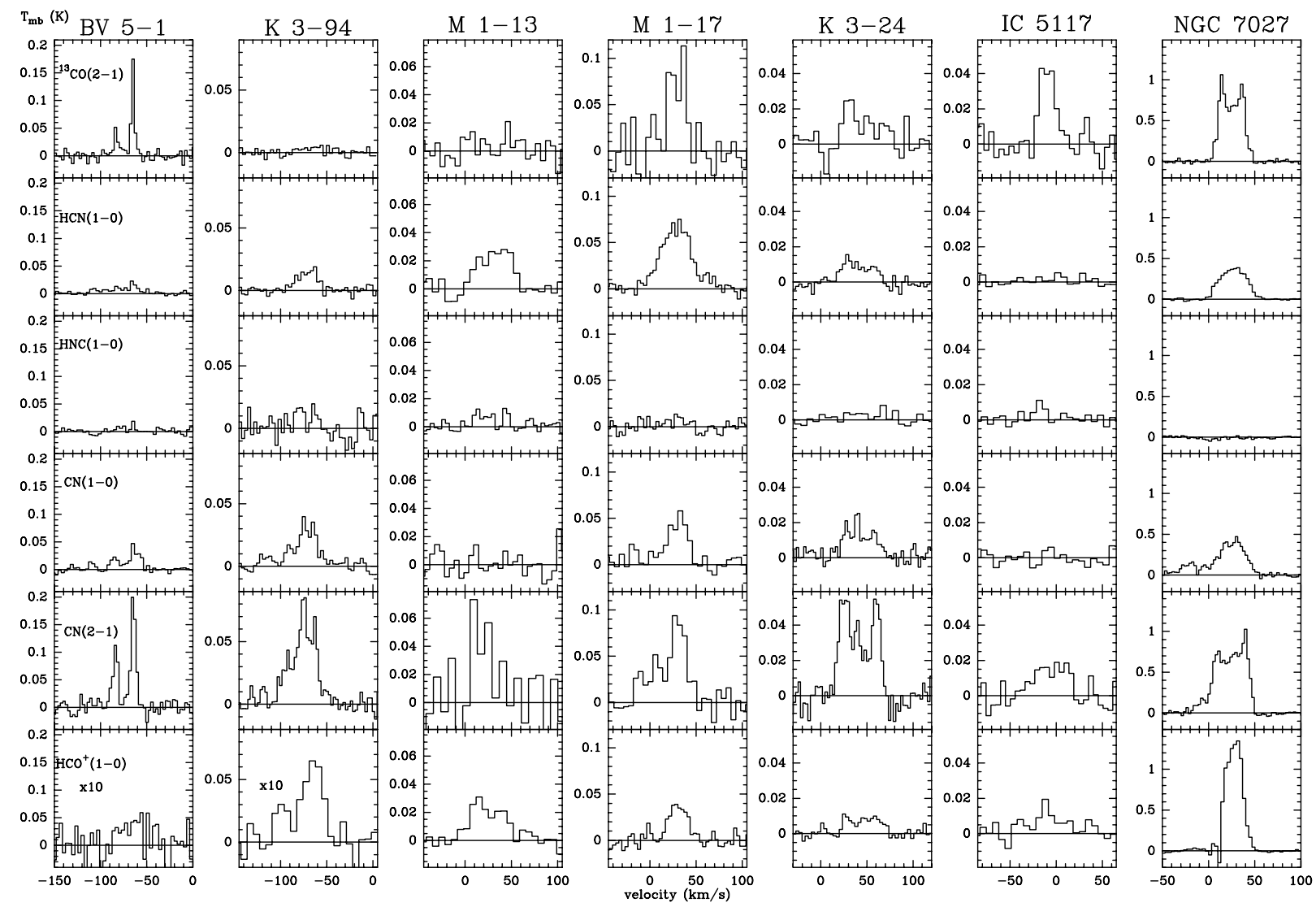

Fig. 1. Spectra of the lines observed in the PN sample. The temperature scale is the same for every line for each $\mathrm{PN}$, except for the $\mathrm{HCO}^{+}$line in PNe BV 5-1 and K 3-94, where the data have been expanded by a factor of 10. The velocity scale of the CN profiles are calculated with respect to the frequency of the strongest component of both lines $\left(v_{0}=113.490945 \mathrm{GHz}\right.$ for the $\mathrm{CN}(1-0)$ line and $v_{0}=226.874766 \mathrm{GHz}$ for the $\mathrm{CN}(2-1)$ line). Some of the data have been smoothed for display purposes in the present figure (line intensities reported in Table 2 have been measured at the original resolution).

2-1 observations provide higher resolution. It should be pointed out that the hyperfine structure of the $\mathrm{CN}$ spectrum may introduce some additional uncertainties in the estimates of column densities. This is because of the possible anomalous excitation through near-infrared pumping which can produce weak masers (Bachiller et al. 1997b). Unfortunately, the separation of the different hyperfine lines is very difficult in the case of our nebulae because of the complex kinematic structure of the line profiles, which often include 2 or 3 components at close velocities (see for instance the $\mathrm{CO}$ profiles in Huggins et al. 1996 and Josselin et al. 2000). We thus simply assumed that the hyperfine line components within a fine group presented standard LTE ratios. The resulting column density estimates for all observed molecules are given in Table 3.

For all the observed species, in all objects other than NGC 7027, the column densities are relatively small which is an indication that the hypothesis of optically thin emission is probably appropriate. Even in NGC 7027, the large column densities may not be incompatible with optically thin emission (Hasegawa \& Kwok 2001). However, the assumptions of LTE and uniform excitation temperatures are much more questionable. In fact, all the observed species but ${ }^{13} \mathrm{CO}$ have high dipole moments ( $\gtrsim 1.5$ Debye) and are thus expected to present subthermal populations. 
Table 2. Integrated intensities of the observed lines, in main-beam scale. Intensities are in $\mathrm{K} \mathrm{km} \mathrm{s}^{-1}$ and $1 \mathrm{rms}$ errors are given in parenthesis. The quoted values for the $\mathrm{CN}$ lines refer to the high frequency fine-structure groups $J=3 / 2 \rightarrow 1 / 2$ for the $\mathrm{CN}(1-0)$ line and $J=5 / 2 \rightarrow 3 / 2$ for the $\mathrm{CN}(2-1)$ line.

\begin{tabular}{|c|c|c|c|c|c|c|c|c|c|c|c|c|c|c|}
\hline \multirow{2}{*}{$\frac{\text { Name }}{\text { BV 5-1 }}$} & \multicolumn{2}{|c|}{${ }^{13} \mathrm{CO}(2-1)$} & \multicolumn{2}{|c|}{$\mathrm{HCN}(1-0)$} & \multicolumn{2}{|c|}{$\mathrm{HNC}(1-0)$} & \multicolumn{2}{|c|}{$\mathrm{CN}(N=1-0)$} & \multicolumn{2}{|c|}{$\mathrm{CN}(N=2-1)$} & \multicolumn{2}{|c|}{$\mathrm{HCO}^{+}(1-0)$} & \multicolumn{2}{|c|}{$\mathrm{N}_{2} \mathrm{H}^{+}(1-0)$} \\
\hline & 1.10 & $(0.05)$ & 0.54 & $(0.02)$ & & $(0.03)$ & 0.77 & $(0.03)$ & 2.22 & $(0.08)$ & 0.12 & $(0.02)$ & & $(0.03)$ \\
\hline K 3-94 & & $(0.15)$ & 0.41 & $(0.03)$ & $\sim 0.35$ & (0.09) & 0.96 & $(0.05)$ & 3.54 & $(0.10)$ & 0.18 & $(0.02)$ & & $(0.04)$ \\
\hline M 1-13 & & $(0.2)$ & 1.16 & $(0.07)$ & $\sim 0.30$ & $(0.07)$ & & $(0.2)$ & 2.16 & $(0.3)$ & 0.95 & $(0.07)$ & & $(0.05)$ \\
\hline M 1-17 & 2.49 & $(0.3)$ & 2.77 & $(0.11)$ & $\sim 0.36$ & $(0.11)$ & 1.39 & $(0.2)$ & 2.77 & $(0.6)$ & 1.18 & $(0.08)$ & & $(0.2)$ \\
\hline K 3-24 & $\sim 0.66$ & $(0.11)$ & 0.58 & $(0.02)$ & & $(0.02)$ & 0.61 & $(0.05)$ & 3.58 & $(0.12)$ & 0.42 & $(0.02)$ & & $(0.01)$ \\
\hline IC 5117 & 0.85 & $(0.11)$ & & $(0.03)$ & $\sim 0.15$ & $(0.04)$ & & $(0.03)$ & 0.81 & $(0.08)$ & 0.45 & $(0.07)$ & & $(0.06)$ \\
\hline NGC 7027 & 26.30 & $(0.2)$ & 12.56 & $(0.2)$ & & $(0.2)$ & 19.15 & $(0.3)$ & 45.49 & $(0.8)$ & 29.78 & $(0.4)$ & 1.74 & $(0.06)$ \\
\hline
\end{tabular}

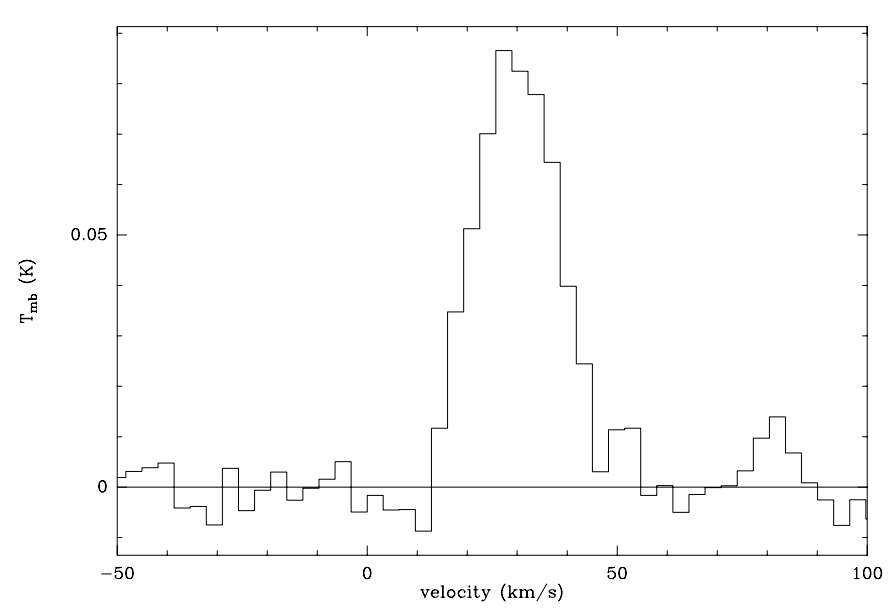

Fig. 2. Spectrum of the $\mathrm{N}_{2} \mathrm{H}^{+}(1-0)$ line towards NGC 7027.

In order to study the evolution of the abundances in the different nebulae, we thus decided to use ratios with respect to HCN (which was detected in all nebulae but IC 5117). Since $\mathrm{HNC}, \mathrm{CN}$ and $\mathrm{HCO}^{+}$have high dipole moments similar to that of HCN, non-LTE effects on the column densities should be of the same order of magnitude for these species so the column density ratios should be less affected by these uncertainties. Furthermore, Hasegawa \& Kwok (2001) found that HCN, $\mathrm{CN}$ and $\mathrm{HCO}^{+}$emission regions have similar sizes in the case of NGC 7027, while CO emission is more spatially extended. Beam dilution effects should thus be less important when comparing emission from the observed species but CO. Figure 3 displays the values of the $\mathrm{HNC}, \mathrm{CN}$ and $\mathrm{HCO}^{+}$column densities with respect to that of $\mathrm{HCN}$. The column density ratios are plotted as a function of the molecular to ionized mass ratio $\left(M_{\mathrm{m}} / M_{\mathrm{i}}\right)$. Data from Bachiller et al. (1997a) for CRL 2688, a typical post-AGB star and the youngest object in their sample, and NGC 7293 (Helix, their oldest PN) have been added, in order to stress the evolution of the abundances. Concerning CRL 2688, one should keep in mind that this object is too young for ionized gas to be present, so its abscissa is arbitrary. Furthermore, its HCN line is optically thick so the relative abundances should be considered as upper limits.

Since, as mentioned before, $M_{\mathrm{m}} / M_{\mathrm{i}}$ is a good indicator of the evolutionary state of the nebula, the abscissas in Fig. 3 can be considered as a time axis, and the lines connecting the values of the column density ratios should reflect the evolution of the abundance ratios. It is interesting to note some general trends in the abundance ratios. The most striking point is the dramatic increase in $\mathrm{HCO}^{+}$abundance relative to $\mathrm{HCN}$ from post-AGB stars to $\mathrm{PNe}$, by more than two orders of magnitude. A secondorder noticeable effect is the slight decrease of the $\mathrm{HCO}^{+} / \mathrm{HCN}$ ratio from rather young $\mathrm{PNe}\left(M_{\mathrm{m}} / M_{\mathrm{i}} \sim 10\right)$ to older ones. The $\mathrm{CN}$ abundance relative to $\mathrm{HCN}$ is observed to increase by a factor of about 20 from the youngest objects in the sample (NGC 7027 and M 1-17) to the oldest ones $\left(M_{\mathrm{m}} / M_{\mathrm{i}} \sim 0.2\right)$. We remind here that the rather high value found for CRL 2688 must be attributed to an under-estimation of the HCN column density. The increase in the $\mathrm{CN}$ abundance occurs essentially during the early stages of the $\mathrm{PN}$ evolution, the $\mathrm{CN} / \mathrm{HCN}$ ratio becomes rather constant after then. The $\mathrm{HNC} / \mathrm{HCN}$ column density ratio does not present a very clear tendency with age. But it should be pointed out that the HNC line was only tentatively detected in some of the objects, and that more sensitive HNC observations are needed before drawing any firm conclusion. Nevertheless, this ratio seems again to be relatively lower in the two youngest objects $(\mathrm{HNC} / \mathrm{HCN} \lesssim 0.2)$ than in some more evolved ones ( $\mathrm{HNC} / \mathrm{HCN}$ up to $\sim 1)$. These values are in the range of those previously observed in other nebulae (Bachiller et al. 1997a) and estimated theoretically (Ali et al. 2001).

The examination of Fig. 3 also gives information about the evolutionary stages of the nebulae. For instance, M 1-17, which was previously suspected to be a young PN because of the presence of wide wings in its spectrum (Bachiller et al. 1991), presents a high $M_{\mathrm{m}} / M_{\mathrm{i}}$ ratio. This, together with its molecular abundances (see Fig. 3), confirms now that M 1-17 is a very young object, similar in many respects to NGC 7027. On the other hand, BV 5-1, K 3-24 and K 3-94 seem to be at a well advanced evolutionary stage, close to that of NGC 7293.

A remarkable exception among the observed behaviours is the case of IC 5117, the only nebula not detected the in $\mathrm{HCN}$ 1-0 line, and with rather weak $\mathrm{CN}$ emission (only the CN 2-1 line was detected), which seems typical of an oxygenrich chemistry, whereas ionized gas in IC 5117 appears carbonrich $(\mathrm{C} / \mathrm{O} \sim 1.3$, Hyung et al. 2001). A C/O ratio $>1$ is consistent with the idea that massive PNe as those in our sample result from the evolution of stars with intermediate masses $\left(M \gtrsim 3 M_{\odot}\right)$ and thus have experienced the 3rd dredgeup, which makes them carbon-rich. However the determination of the carbon abundance in IC 5117 is very uncertain. 
Table 3. Column densities (in $\mathrm{cm}^{-2}$ ) derived from observed line intensities given in Table 2 (see Sect. 4.1 for details).

\begin{tabular}{lrrrrr}
\hline \hline Name & $N\left({ }^{13} \mathrm{CO}\right)$ & $N(\mathrm{HCN})$ & $N(\mathrm{HNC})$ & $N(\mathrm{CN})$ & $N\left(\mathrm{HCO}^{+}\right)$ \\
\hline BV 5-1 & $3.9 \times 10^{14}$ & $1.3 \times 10^{12}$ & $\lesssim 2.4 \times 10^{11}$ & $3.0 \times 10^{12}$ & $1.7 \times 10^{11}$ \\
K 3-94 & $\lesssim 1.6 \times 10^{14}$ & $1.0 \times 10^{12}$ & $\sim 9.1 \times 10^{11}$ & $4.7 \times 10^{12}$ & $2.6 \times 10^{11}$ \\
M 1-13 & $\lesssim 2.1 \times 10^{14}$ & $2.9 \times 10^{12}$ & $\sim 7.9 \times 10^{11}$ & $2.9 \times 10^{12}$ & $1.4 \times 10^{12}$ \\
M 1-17 & $8.9 \times 10^{14}$ & $6.9 \times 10^{12}$ & $\sim 9.4 \times 10^{11}$ & $3.7 \times 10^{12}$ & $1.7 \times 10^{12}$ \\
K 3-24 & $\sim 2.4 \times 10^{13}$ & $1.4 \times 10^{12}$ & $\lesssim 4.2 \times 10^{11}$ & $4.8 \times 10^{12}$ & $6.0 \times 10^{11}$ \\
IC 5117 & $3.0 \times 10^{14}$ & $\lesssim 2.2 \times 10^{11}$ & $\sim 3.8 \times 10^{11}$ & $1.1 \times 10^{12}$ & $5.5 \times 10^{11}$ \\
NGC 7027 & $9.4 \times 10^{15}$ & $3.1 \times 10^{13}$ & $\lesssim 9.3 \times 10^{12}$ & $6.1 \times 10^{13}$ & $4.3 \times 10^{13}$ \\
\hline
\end{tabular}

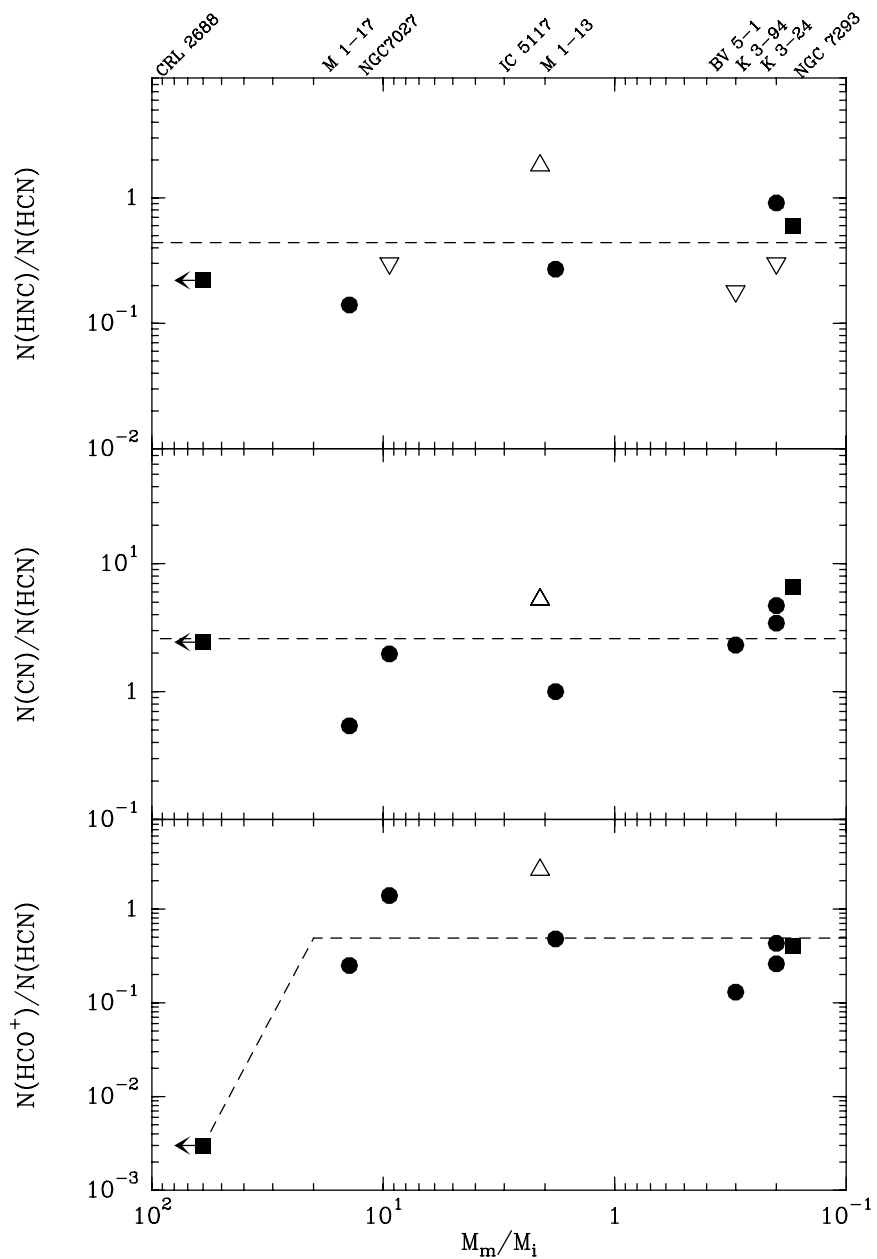

Fig. 3. Abundances of $\mathrm{HNC}, \mathrm{CN}$ and $\mathrm{HCO}^{+}$relative to $\mathrm{HCN}$ as a function of the molecular to ionized mass ratio. The ratio is plotted with a filled circle when both molecules are detected and with an empty triangle if one of the molecules is not detected, the orientation of the triangle indicating if the limit is a lower or an upper one. Data for CRL 2688 and NGC 7293, indicated by squares, are from Bachiller et al. (1997a). Horizontal dashed lines indicate the average of the relative abundances within our sample.

Furthermore, the existence of massive oxygen-rich $\mathrm{PNe}$ is not excluded, because of hot-bottom burning which could convert ${ }^{12} \mathrm{C}$ into ${ }^{13} \mathrm{C}$ and ${ }^{14} \mathrm{~N}$, decreasing the relative abundance of carbon (Renzini \& Voli 1981). One could also think about a chemical stratification in the nebula, as the molecular gas corresponds to mass ejection prior to that traced by ionized gas. Additional observations are needed to understand this peculiar PN.

\subsection{Carbon isotopic ratio}

In the case of PNe, the observed $I\left({ }^{12} \mathrm{CO}(2-1)\right) / I\left({ }^{13} \mathrm{CO}(2-1)\right)$ intensity ratio is expected to be a good indicator of the isotopic ${ }^{12} \mathrm{C} /{ }^{13} \mathrm{C}$ abundance ratio (see Palla et al. 2000). In fact, the intensity ratio equals the abundance ratio when the following assumptions are accomplished : (i) the ${ }^{12} \mathrm{CO}(2-1)$ and ${ }^{13} \mathrm{CO}(2-1)$ rotational levels are thermalized, (ii) the ${ }^{12} \mathrm{CO}(2-1)$ and ${ }^{13} \mathrm{CO}(2-1)$ are not very optically thick, and (iii) the beam filling factor is similar for both lines. Since the dipole moment of CO is small ( $\sim$.11 Debye), the first assumption is very plausible. In addition, saturation effects are expected to be negligible for most evolved PNe, since the $I(\mathrm{CO}(2-1)) / I(\mathrm{CO}(1-0))$ ratio suggests optically thin emission (Huggins et al. 1996; Josselin et al. 2000). But this may not the case for the youngest $\mathrm{PNe}$ (such as NGC 7027 and M 1-17), for which the ${ }^{12} \mathrm{CO}$ lines are probably optically thick; isotopic ratios for these objects should thus been considered as lower limits.

The validity of the hypothesis of equal beam filling depends directly on the spatial distribution of the $\mathrm{CO}$ isotopomers, and thus on selective processes of formation and destruction of the molecules. Two types of reactions may be important to determine the $\mathrm{CO}$ isotopomer ratio: selective photodissociation, ${ }^{13} \mathrm{CO}$ being more easily photodissociated, and chemical fractionation through the reaction

${ }^{12} \mathrm{CO}+{ }^{13} \mathrm{C}^{+} \rightarrow{ }^{13} \mathrm{CO}+{ }^{12} \mathrm{C}^{+}$

But these effects are generally thought to compensate each other (see Mamon et al. 1988 for a more detailed discussion). In summary, we believe that for most of the PNe discussed here, ${ }^{12} \mathrm{C} /{ }^{13} \mathrm{C}=I\left({ }^{12} \mathrm{CO}(2-1)\right) / I\left({ }^{13} \mathrm{CO}(2-1)\right)$. The isotopomer ratios resulting from the observations are summarized in Table 4.

We find ratio values in the range $10 \lesssim{ }^{12} \mathrm{CO} /{ }^{13} \mathrm{CO} \lesssim 40$. The ${ }^{12} \mathrm{C} /{ }^{13} \mathrm{C}$ ratio was recently measured in some additional PNe by Palla et al. (2000) and Balser et al. (2002), and the values reported by these authors are in general agreement with the ones reported here. These values are below those expected from standard asymptotic giant branch models and suggest non-standard mixing processes. 
Table 4. Carbon isotopic ratios in the PNe of our sample.

\begin{tabular}{lr}
\hline \hline Name & ${ }^{12} \mathrm{C} /{ }^{13} \mathrm{C}$ \\
\hline BV 5-1 & 9 \\
K 3-94 & $\gtrsim 31$ \\
M 1-13 & $\gtrsim 37$ \\
M 1-17 & 27 \\
K 3-24 & 35 \\
IC 5117 & 23 \\
NGC 7027 & 11 \\
\hline
\end{tabular}

We have estimated the mass of the progenitors of BV 5-1 and K 3-94, by assuming relevant stellar parameters taken from the Acker et al. (1992) catalogue, and by following the procedure described in Palla et al. (2000). For M 1-17, IC 5117 and NGC 7027, estimates are provided by Palla et al. (2000). For M 1-13 and K 3-24, the lack of the central star photometry prevents any realistic estimate to be done. Of the studied sample, BV 5-1 and K 3-94 appear to have the most massive progenitors, with $\sim 5.5$ and $\sim 4.0 M_{\odot}$, respectively (we note however that the estimate of the progenitor mass requires the previous estimate of the luminosity of the central star which in turn depends on the choice of the distance, which as discussed above remains quite uncertain). Then, when reported in Fig. 3 of Palla et al., the ${ }^{12} \mathrm{C} /{ }^{13} \mathrm{C}$ isotopic ratio appears consistent with the models they quote. We thus confirm that non-standard mixing processes should thus be important only in low-mass stars, as suggested by Charbonnel (1995).

\section{Discussion}

We next discuss the chemical processes which must be relevant in the case of the molecules observed here by strengthening the differences between the conditions prevailing in PNe from those of the interstellar medium. Special attention is devoted to the clumpiness of the neutral PN envelopes since clumping is expected to play a key role in the preservation and evolution of the molecular species.

\subsection{The survival of molecules in PNe}

The compact PNe discussed here sample the evolution of the chemistry from the younger objects to rather developed PNe. An interesting result from our observations is the continuity of the molecular content found for PNe with very different evolutionary status and morphologies. In particular, N-bearing molecules are present at all the sampled evolutionary stages. This finding is in contrast with the recent suggestion made by Hasegawa et al. (2000) that most molecules from the AGB remnant (except $\mathrm{H}_{2}$ and $\mathrm{CO}$ ) are completely destroyed and then reformed at the PN stage. Our observations suggest on the contrary that most molecular species survive during the transition from the AGB to the PN phase.

An exception to this continuity in the molecular content is provided by the sudden increase in the $\mathrm{HCO}^{+}$abundance. This ion is expected to form in warm PDR-like regions such as the interface between ionized and molecular gas. Even if currently available models fail in reproducing the observed abundance, they clearly show that the abundance is sensitive to the total density (see e.g. Table 3 in Hasegawa \& Kwok 2001). Additional observational probes of these PDR-like regions would be provided by measuring the $\mathrm{CO}^{+}$and $\mathrm{N}_{2} \mathrm{H}^{+}$ abundances, but the corresponding observations would require higher sensitivity than that of the observations presented here.

\subsection{Chemistry of $N$-bearing molecules}

The chemistry of N-bearing molecules has been recently studied by Ali et al. (2001) with a detailed time-dependent chemical model suited to the conditions of evolved PNe, and by Hasegawa et al. (2000) and Hasegawa \& Kwok (2001) for the particular case of NGC 7027. The $\mathrm{CN} / \mathrm{HCN}$ ratio calculated by Hasegawa et al. (2000) is in the range 1.4 to 6.6, depending on the volume hydrogen density and the kinetic temperature, in good agreement with our average value $(\sim 2.6)$. The abundances of $\mathrm{HCN}$ and HNC calculated by Ali et al. (2001) are in general agreement with the observations reported here. However their abundance of $\mathrm{CN}$ is too high by a factor of $\sim 3$.

The chemistries of the three observed molecules ( $\mathrm{HCN}$, $\mathrm{HNC}$ and $\mathrm{CN}$ ) are intimately interconnected. $\mathrm{CN}$ is mainly produced through the photodissociation of $\mathrm{HCN}$ or $\mathrm{HNC}$, both having similar unshielded rates $\left(\sim 1.4 \times 10^{-9} \mathrm{~s}^{-1}\right.$, Le Teuff et al. 2000). It is thus expected that $\mathrm{CN}$ is mainly located in a kind of shell surrounding the $\mathrm{HCN} / \mathrm{HNC}$ regions, where the ambient UV radiation coming from the central star can penetrate to photodissociate $\mathrm{HCN}, \mathrm{HNC}$, and other related compounds. The $\mathrm{CN}$ and $\mathrm{HCN}$ emitting regions are thus not expected to be coincident, and the $\mathrm{CN} / \mathrm{HCN}$ abundance ratio can strongly vary with the position in the nebula. The $\mathrm{CN} / \mathrm{HCN}$ column density ratios provided above must be considered as average values.

One could ask about the potential importance of two body reactions in the chemistry of nitrogen. Reactions such as that of atomic carbon with $\mathrm{NO}$ and $\mathrm{N}_{2}$ or atomic nitrogen with $\mathrm{CH}$ or $\mathrm{CS}$, should have negligible contributions, both because such reactions have comparatively lower rates and the reactants must have very low abundances. For example, atomic carbon is only present in a very thin transition region between molecular gas $(\mathrm{CO})$ and ionized gas $\left(\mathrm{C}^{+}\right)$, as carbon ionization potential and $\mathrm{CO}$ dissociation energy are similar $(\sim 11 \mathrm{eV})$.

One exception may be the reaction

$\mathrm{O}+\mathrm{HCN} \rightarrow \mathrm{CN}+\mathrm{OH}$

as atomic oxygen may be abundant in photodissociation regions. Unfortunately, the abundance of atomic oxygen is not well known in these regions, and it will certainly strongly depend on the carbon-rich or oxygen-rich character of the envelope. If atomic oxygen turned out to be significantly abundant, this reaction could accentuate the production of $\mathrm{CN}$ at the expense of HCN. Nevertheless, the presence of atomic oxygen can also contribute to the destruction of $\mathrm{CN}$ :

$\mathrm{O}+\mathrm{CN} \rightarrow \mathrm{CO}+\mathrm{N}$ or $\mathrm{NO}+\mathrm{C}$.

The relative importance of these processes should be examined in the peculiar case of PN IC 5117 (see Sect. 3.2). 
In principle, $\mathrm{HCN}$ and $\mathrm{HNC}$ can convert to each other through hydrogen exchange reaction

$\mathrm{H}+\mathrm{HNC} \leftrightarrow \mathrm{HCN}+\mathrm{H}$.

However, the forward reaction must be predominant since it has an activation energy four times smaller than the reverse reaction (Talbi et al. 1996). On the other hand, the HNC/HCN ratio is observed to decrease at increasingly high densities in interstellar clouds (Turner et al. 1997). The relatively low $\mathrm{HNC} / \mathrm{HCN}$ ratio observed in $\mathrm{PNe}$ could then be due to the combination of moderate temperatures and densities prevailing in the molecular envelopes.

$\mathrm{HCN}$ and $\mathrm{HNC}$ can indeed react with several molecular ions such as $\mathrm{HCO}^{+}, \mathrm{H}_{3}^{+}, \mathrm{H}_{3} \mathrm{O}^{+}$or $\mathrm{C}_{2} \mathrm{H}^{+}$to form $\mathrm{HCNH}^{+}$, which after dissociative recombination will lead to $\mathrm{CN}, \mathrm{HCN}$ or $\mathrm{HNC}$ in a ratio 2/1/1 (Le Teuff et al. 2000). As the abundance of molecular ions is expected to increase with the state of evolution (as the nebula is being progressively ionized), such reactions are expected to additionally enhance the $\mathrm{CN} / \mathrm{HCN}$ ratio as the evolution proceeds.

\subsection{Molecular ions: $\mathrm{HCO}^{+}$and $\mathrm{N}_{2} \mathrm{H}^{+}$}

As already discussed by Bachiller et al. (1997a), a remarkable result concerning the chemical content of $\mathrm{PNe}$ is the rather high column densities of $\mathrm{HCO}^{+}$. The "standard" path of $\mathrm{HCO}^{+}$ formation which may dominate in dense interstellar clouds invokes the reaction of $\mathrm{H}_{3}^{+}$with $\mathrm{CO}$, and thus requires a high formation rate of $\mathrm{H}_{3}^{+}$through cosmic-ray ionization of $\mathrm{H}_{2}$ (Turner 1995). However the survival of $\mathrm{H}_{3}^{+}$ion is expected to be very short, because of its very effective dissociative recombination (Bachiller et al. 1997a), so this process may not be efficient enough to explain the observed high abundances. It has been pointed out by Ali et al. (2001) that X-ray emission from the central hot star of $\mathrm{PNe}$ may also contribute to a high rate of ionization. In fact, the relatively high X-ray fluxes have been observed in several PNe (e.g. Apparao \& Tarafdar 1989) are believed to be generated from the central star (not from the nebula). The effect of such X-rays on the envelope can be particularly important in the case of compact PNe as these studied here.

An additional way to produce $\mathrm{HCO}^{+}$is through the reaction

$\mathrm{CO}^{+}+\mathrm{H}_{2} \rightarrow \mathrm{HCO}^{+}+\mathrm{H}$

$\mathrm{CO}^{+}$could be formed through charge transfer between $\mathrm{H}^{+}$and $\mathrm{CO}$, via the reaction

$\mathrm{C}^{+}+\mathrm{OH} \rightarrow \mathrm{CO}^{+}+\mathrm{H}$

or by the direct ionization of CO by the means, for instance, of the propagation of an ionizing front into the molecular gas of high CO abundance (Latter et al. 1993). The latter path may be dominant at least in some PNe. The initiation of such ionizing front is one of the characteristics of PPNe (Kwok 1993) and $\mathrm{HCO}^{+}$seems indeed to form essentially during this stage (Bachiller et al. 1997a). Besides the formation of $\mathrm{HCO}^{+}$, the other main destruction process of $\mathrm{CO}^{+}$is dissociative recombination. A column density $N\left(\mathrm{CO}^{+}\right) \gtrsim 10^{12} \mathrm{~cm}^{-2}$ can be expected and is consistent with the detection in NGC 7027 (Latter et al. 1993). A systematic search for this ion in PNe where $\mathrm{HCO}^{+}$emission is intense would confirm this scenario.

$\mathrm{HCO}^{+}$is mainly destroyed by dissociative recombination with electrons. These chemical processes are included in the detailed model of Ali et al. (2001) that leads to an abundance ratio $\mathrm{HCO}^{+} / \mathrm{HCN} \sim 0.1$, in reasonable agreement with the less evolved PNe of our sample, but significantly lower (by a factor of 5 to 10) that the values found in $\mathrm{PNe}$ at intermediate stage of evolution (see also Bachiller et al. 1997a). The observed trend in the evolution of $\mathrm{HCO}^{+}$abundance seems to imply an increase in electron density during the late stages of PN evolution. Hasegawa \& Kwok (2001) found that in NGC 7027 about $90 \%$ of carbon, which is a major source of electrons, is in the form of $\mathrm{C}^{+}$. Carbon with higher ionization degree $\left(\mathrm{C}^{2+}\right.$ and $\left.\mathrm{C}^{3+}\right)$ is commonly observed toward more evolved PNe. An increase in the destruction process of $\mathrm{HCO}^{+}$during the last evolutionary stages is thus expected and consistent with our observations. The abundance of $\mathrm{HCO}^{+}$estimated by Hasegawa et al. (2000) for NGC 7027 is in general agreement with our observations, but it assumes that it is formed in gas at a temperature of $\sim 800 \mathrm{~K}$. Observations of spectral lines from higher J levels (at higher frequencies) would be useful to estimate the gas physical conditions.

In molecular clouds, $\mathrm{N}_{2} \mathrm{H}^{+}$is often thought to be associated with $\mathrm{HCO}^{+}$, since it is essentially formed through

$\mathrm{H}_{3}^{+}+\mathrm{N}_{2} \rightarrow \mathrm{N}_{2} \mathrm{H}^{+}+\mathrm{H}$.

But contrary to $\mathrm{HCO}^{+}$, no important alternative formation is expected. The abundance of $\mathrm{N}_{2} \mathrm{H}^{+}$was however not reported in the models of Ali et al. (2001). The lack of other significant paths of formation, together with its high molecular dipole moment ( 3.4 Debye) explains why we only detected this ion in NGC 7027. Assuming similar line intensity ratios in the other $\mathrm{PNe}$, one needs to reach a rms $\lesssim 0.5 \mathrm{mK}$ to allow its detection, far below the limit we obtained. A deeper search for this ion would be interesting to examine any correlation with the temperature of the central star and in particular with its X-ray flux (when $T_{\star} \gtrsim 10^{5} \mathrm{~K}$ ).

\subsection{Clumpiness and PDR-like regions}

Beside the evolutionary trends described above, a rather large dispersion of abundance ratios is observed for different values of $M_{\mathrm{m}} / M_{\mathrm{i}}$. Such scatter may be, at least partly, attributed to the the number and density of the clumps, which are expected to vary from one PN to another. Indeed these variations should act on the observed abundances by two ways. First, the degree of clumpiness (filling factor) can be different for different molecules in the highly fragmented envelopes of the more evolved objects such as BV 5-1 (Josselin \& Bachiller 2001). Second, the survival of the molecules is expected to strongly depend on the number and density of the clumps which determine the shielding of dissociating UV radiation from the central star. Indeed, high resolution maps of the molecular envelopes of evolved PNe show that the molecular material is always confined within the ionized nebula (see e.g. Speck et al. 2002 for the Helix nebula and Josselin \& Bachiller 2001 
for BV 5-1), in contradiction with the simple image of an initially neutral homogeneous nebula being gradually ionized ("Strömgren sphere"). As confirmed by direct observations of some individual dense clumps in the Helix (Huggins et al. 1992, 2002), the molecules are in fact concentrated within dense blobs which reach a state of approximate dynamical equilibrium with the surrounding ionized material, and whose evolution is determined by their density, i.e. by their capability to be efficiently shielded from the ambient UV radiation. Any consistent chemical model of the neutral matter in PNe should thus take into account this clumpy structure which seems to be an essential ingredient of PNe.

\section{Conclusions}

The presence of molecular gas in compact $\mathrm{PNe}$ provides a unique opportunity to examine a rapidly evolving chemistry in a peculiar medium which is subjected to strong radiation fields. The observations reported in this paper show that some species such as $\mathrm{HCO}^{+}$and $\mathrm{CN}$ are particularly abundant compared to envelopes around AGB stars or even to interstellar clouds. The chemical composition of the molecular envelopes in these compact PNe appears somewhat intermediate between the composition of proto-PNe (such as CRL 2688 or CRL 618) and well evolved PNe (such as the Ring, M4-9, or the Helix).

The molecular abundances are well explained, in general terms, by time-dependent gas-phase chemical models in which the ionization rate is enhanced by several orders of magnitude with respect to the average interstellar value (Ali et al. 2001; Hasegawa et al. 2000; Hasegawa \& Kwok 2001). Thus, our observations confirm that the chemistry in the neutral shells of $\mathrm{PNe}$ is essentially governed by the high energy radiation from the hot central stars. Shocks due to fast winds which develop during the formation of the PN can also be important, but mainly during the proto-PN stage and in the regions where the jets impact on the envelope. The complexity of the chemical processes is increased by numerous factors linked to the properties of the central star and the geometry and degree of clumpiness of the envelope. The survival of molecules can only occur in well protected dense clumps, and a rich photochemistry very likely takes place at the surfaces of these PDRlike regions.

Although the main processes governing the chemistry in the neutral envelopes of PNe seem well identified, some significant discrepancies with the models remain to be understood. For instance the calculated $\mathrm{CN} / \mathrm{HCN}$ ratio is a factor of $\sim 5$ higher than the observed value, and the observed $\mathrm{HCO}^{+}$ abundances tend to be higher than those predicted by the models. In addition, as discussed by Hasegawa et al. (2000), some of the molecules observed in evolved PNe (such as the Ring or the Helix) could not be direct remnants from the AGB envelope, but newly formed species, this is the case of $\mathrm{HCO}^{+}$. However, the observations suggest that there is a continuity in the chemical composition from the proto-PN phase to the full photodissociation of the envelope, and that molecules like $\mathrm{CO}$ and $\mathrm{HCN}$ are progressively photo-dissociated.

Additional observations are now required to push up our understanding of this chemistry. The search for species such as
$\mathrm{CO}^{+}$would be useful to confirm the chemical networks invoked to explain the observed abundances.

Acknowledgements. The authors acknowledge many interesting discussions and collaborative work on PNe with Drs. P. Cox, T., Forveille, and P. J. Huggins. RB acknowledges warm hospitality during a stay at GRAAL (University of Montpellier) during which part of this manuscript was written. EJ acknowledges partial financial support from french CNRS grant ATIPE and RB from spanish MCYT grant AYA2000-927.

\section{References}

Acker, A., Ochsenbein, F., Sternholm, B., et al. 1992, Strasbourg-ESO Catalogue of Galactic Planetary Nebulae

Ali, A., Shalabeia, O. M., El-Nawawy, M. S., \& Millar, T. J. 2001, MNRAS, 325, 881

Apparao, K. M. V., \& Tarafdar, S. P. 1989, ApJ, 344, 826

Bachiller, R., Forveille, T., Huggins, P. J., \& Cox, P. 1997a, A\&A, 324,1123

Bachiller, R., Fuente, A., Bujarrabal, V., et al. 1997b, A\&A, 319, 235

Bachiller, R., Huggins, P. J., Cox, P., \& Forveille, T. 1991, A\&A, 247, 525

Balser, D. S., McMullin, J. P., \& Wilson, T. L. 2002, ApJ, 572, 326

Black, J. H. 1978, ApJ, 222, 125

Bujarrabal, V., Gomez-Gonzalez, J., Bachiller, R., \& Martin-Pintado, J. 1988, A\&A, 204, 242

Burton, W. B. 1974, in Galactic and extra-galactic radio astronomy, ed. G. Verschuur, \& K. I. Kellermann (Springer)

Cernicharo, J., Heras, A. M., Tielens, A. G. G. M., et al. 2001, ApJ, 546, L123

Charbonnel, C. 1995, ApJ, 453, L41

Cox, P., Bachiller, R., Huggins, P. J., et al. 1993, IAU Symp., 155, 227

Cox, P., Omont, A., Huggins, P. J., et al. 1992, A\&A, 266, 420

Gathier, R. 1987, A\&AS, 71, 245

Hasegawa, T., \& Kwok, S. 2001, ApJ, 562, 824

Hasegawa, T., Volk, K., \& Kwok, S. 2000, ApJ, 532, 994

Howe, D. A., Hartquist, T. W., \& Williams, D. A. 1994, MNRAS, 271, 811

Howe, D. A., Millar, T. J., \& Williams, D. A. 1992, MNRAS, 255, 217

Huggins, P. J., Bachiller, R., Cox, P., \& Forveille, T. 1992, ApJ, 401, L43

Huggins, P. J., Bachiller, R., Cox, P., \& Forveille, T. 1996, A\&A, 315, 284

Huggins, P. J., Forveille, T., Bachiller, R., et al. 2002, ApJ, 573, L55

Hyung, S., Aller, L. H., Feibelman, W. A., \& Lee, S.-J. 2001, ApJ, 563, 889

Josselin, E., Bachiller, R., Manchado, R., \& Guerrero, M. A. 2000, A\&A, 353, 363

Josselin, E., \& Bachiller, R. 2001, A\&A, 376, 484

Kastner, J. H., Weintraub, D. A., Gatley, I., et al. 1996, ApJ, 462, 777

Kwok, S. 1993, ARA\&A, 31, 63

Latter, W. B., Walker, C. K., \& Maloney, P. R. 1993, ApJ, 419, L97

Le Teuff, Y. H., Millar, T. J., \& Markwick, A. J. 2000, A\&AS, 146, 157

Mamon, G., Glassgold, A. E., \& Huggins, P. J. 1988, ApJ, 328, 797

Palla, F., Bachiller, R., Stanghellini, L., et al. 1998, A\&A, 355, 69

Renzini, A., \& Voli, M. 1981, A\&A, 94, 175

Sánchez Contreras, C., Bujarrabal, V., \& Alcolea, J. 1997, A\&A, 327, 689

Speck, A. K., Meixner, M., Fong, D., et al. 2002, AJ, 123, 346

Talbi, D., Ellinger, Y., \& Herbst, E. 1996, A\&A, 314, 688

Turner, B. E. 1995, ApJ, 449, 635

Turner, B. E., Pirogov, L., \& Minh, Y. C. 1997, ApJ, 483, 235 